\title{
El uso del podcasting como herramienta de la identidad corporativa en la campaña del 90 aniversario de lberia. El caso del podcast Aerolínea Momentos.
}

\section{The use of podcasting to communicate the corporate identity in the Iberia's 90th anniversary campaign. The case of the podcast Aerolínea Momentos.}

\author{
Autor: \\ Dr. Toni Sellas \\ Orcid: http://orcid.org/0000-0003-2653-0547 \\ toni.sellas@uvic.cat \\ Professor Agregat \\ Departament de Comunicació, Facultat d'Empresa i Comunicació \\ Universitat de Vic - Universitat Central de Catalunya
}

\section{Resumen}

La incorporación de las tecnologías de la información y la comunicación en las relaciones públicas es un factor disruptivo que altera las dinámicas entre organizaciones, profesionales y públicos. En este contexto, el podcasting presenta algunas singularidades. No obstante, su uso en las estrategias corporativas es todavía incipiente. Este artículo estudia el caso del podcast Aerolínea Momentos, coproducido por Podium Podcast e Iberia. El objetivo es explicar la integración del podcast en la campaña de celebración del 90 aniversario de la compañía aérea, exponer sus características distintivas y analizar su encaje con la identidad corporativa de la empresa. Se trata de un estudio cualitativo, basado una investigación empírica secundaria a partir de fuentes documentales seleccionadas por su credibilidad, autenticidad y representatividad, y en un análisis de contenido de los nueve episodios del podcast mediante PodCred Framework, marco de análisis contrastado por investigaciones internacionales

\section{Abstract}

The incorporation of information and communication technologies in public relations is a disruptive movement that modifies the dynamics between organizations, professionals and publics. In this context, podcasting has some particular characteristics. However, its use in corporate strategies is still incipient. This article studies the case of Aerolínea Momentos, a podcast coproduced by Podium Podcast and Iberia. The main objectives of this article are to explain the podcast integration in the Iberia's campaign planned to celebrate its $90^{\text {th }}$ anniversary, to expose the distinctive features of this audio fiction and to analyze how it fits with the Iberia's corporate identity. This case study follows a qualitative methodology. On the one hand, it is based on a secondary empirical research using corporate and journalistic documents selected due to their credibility, authenticity and representativeness. On the other hand, it uses a content analysis of the nine episodes of the podcast using the PodCred Framework, 
para la valoración del potencial e interés de los podcasts. El estudio de caso muestra un buen encaje del podcast en la estrategia de la organización. Paralelamente, Aerolínea Momentos cumple con la mayoría de los indicadores de calidad del PodCred Framework. La especialización de los profesionales que intervienen en la producción resulta clave para un resultado óptimo como branded podcast de ficción. En definitiva, Aerolínea Momentos es un caso de éxito por la confluencia de una sólida estrategia comunicativa desde la dirección de comunicación de lberia y una producción profesional y atractiva a cargo de especialistas en la ficción sonora en Podium Podcast.

Palabras clave: Podcasting, branded podcast, ficción sonora, identidad corporativa, relaciones públicas. a scheme of analysis contrasted by international researches for the assessment of the potential and interest of podcasts. The case study shows how the podcast fits well with the corporate strategy. In addition, Aerolínea Momentos meets most of the quality indicators of the PodCred Framework. The specialization of the team involved in the production is a key factor to get an optimal result as a branded podcast. In short, Aerolínea Momentos is successful because of the confluence of a strong communication strategy from Iberia and an excellent production by specialists in audio fiction from Podium Podcast.

Keywords: Podcasting, branded podcast, audio fiction, corporate identity, public relations.

\section{Introducción}

La incorporación de tecnologías basadas en Internet ha impactado en la relación de las organizaciones con sus públicos (Duhé, 2007), aunque su utilización estratégica todavía refleja carencias (Taylor y Kent, 2010). En este contexto, el podcasting presenta algunas singularidades como alternativa o complemento a los medios más habituales. No obstante, su uso en las organizaciones es todavía incipiente. Este artículo pretende contribuir al conocimiento de las características y potencial del podcasting en el ámbito de las relaciones públicas, mediante el análisis de la utilización de los podcasts en la campaña del 90 aniversario de la compañía aérea Iberia.

\subsection{Tecnología y web social en las relaciones públicas}

La tecnología ha impactado como factor disruptivo en las dinámicas entre organizaciones, profesionales y públicos. Las organizaciones se han ido adaptando a un nuevo terreno de juego en el que los públicos desempeñan un rol activo. El contexto requiere un planteamiento dialógico y ofrece muchas oportunidades, a la vez que exige un mayor esfuerzo para conocer y comprender los intereses, las preocupaciones y los comportamientos de los públicos (Estanyol, 2012). El papel de las TIC y la utilización de los medios sociales en la práctica profesional han sido objeto de diversos análisis, con resultados dispares. Tras una amplia revisión de la literatura científica, McCalllister-Spooner (2009) concluía que la promesa de unas relaciones públicas en Internet basadas en el diálogo con los públicos distaba mucho de ser una realidad. Taylor y Kent (2010) iban más allá, al afirmar que no existían pruebas empíricas del poder de los medios sociales en las relaciones públicas. 
Otros estudios más recientes han situado el foco en la investigación académica. Verčič, Verčič y Siramesh (2015) llevan a cabo una extensa revisión de artículos publicados en Public Relations Review, desde su primer número de 1975, focalizado en 155 artículos que tratan del vínculo de las relaciones públicas con las TIC y lo que denominan digital, social and mobile (DSM) media. El resultado refleja un crecimiento desmesurado del campo, pero con un enfoque centrado principalmente en la relación con los medios. Por el contrario, critican un esfuerzo escaso para definir y entender a los públicos, así como una total desatención a cuestiones relevantes como la brecha digital, la privacidad o la disolución de las barreras entre relaciones públicas, publicidad y periodismo. El artículo también revela que social media es el término más mencionado en el título de los artículos analizados, con 30 apariciones. En el extremo contrario, solo aparece una referencia a términos como smartphones, social networking, nanoblogging o podcasts, entre otros.

Duhé (2015) revisa 321 artículos publicados entre 1981 y 2014 en seis revistas académicas del ámbito. Su análisis constata un predominio de los artículos sobre la aplicación de las tecnologías o las percepciones de los profesionales, y una atención menor a cuestiones como la usabilidad o los riesgos éticos y legales de los nuevos medios. En cuanto a la aplicación de los nuevos medios, solo un artículo de los 321 analizados versa sobre el podcasting. Por su parte, Fernández-Quijada y Ramos-Serrano realizan una aproximación sociocéntrica a la tecnología, en un volumen que proporciona claves "para contrarrestar el discurso triunfalista de algunos profesionales, medios y blogueros" (2014, p. 26).

\subsection{De la radio corporativa al branded podcast}

La presencia del audio como soporte a la comunicación de las organizaciones no es nueva, especialmente en la publicidad. Sin embargo, como señala Piñeiro-Otero (2015, p. 678), "si damos un paso más y nos referimos al audiobranding como un empleo estratégico del sonido, que se inserta en una concepción global e integrada de la marca, debemos señalar lo novedoso del fenómeno". La autora propone una tipología que diferencia tres bloques: a) publicidad (música anuncios, jingle); b) identidad de marca (logo sonoro, claim sonoro, voz de marca/ corporativa, canción de marca/corporativa, sound design de producto); y c) branded content (eventos musicales, plataformas musicales 2.0 , radios corporativas).

Prata y Cordeiro (2017, p. 78) definen la radio corporativa como "una estación hecha a la medida para una empresa en particular, siguiendo los preceptos de su cultura organizacional". Por su parte, Peinado-Miguel, Rodríguez-Barba y Rodríguez-Pallares analizan los diferentes tipos de radio corporativa - staff radio, radio institucional, brand radio e in store radio (Sánchez, 2010) - y concluyen que "la base de la radio corporativa es el podcast, [...] cuyo contenido ha de ser atemporal, de interés, de comercialización perfilada y con una distribución multiplataforma" (2017, p. 162).

La evolución del audio corporativo pasa por la integración del podcasting, una opción singular pero todavía menor en las relaciones públicas:

[...] del mimo modo que organizaciones y agencias se han preocupado por formar o contratar especialistas en redes sociales, deberían pensar en profesionales con el co nocimiento y las competencias necesarios para ocuparse de los podcasts corporativos. 
El podcasting es diferente al resto de los medios sociales, y en tiempos de saturación informativa se hace necesario probar fórmulas creativas para las relaciones con los públicos (Sellas, 2014, p. 211).

El uso de los podcasts empieza a extenderse en las estrategias corporativas (Daniels, 2017). Técnicamente, el podcasting es el resultado de la suma del audio digital y la sindicación web, que permite subscribirse a nuevo contenido que se descarga automáticamente y se puede reproducir posteriormente en diversos tipos de dispositivos. Esta tecnología permite al oyente decidir cuándo, dónde y cómo escucha el contenido. Desde el punto de vista creativo, con unos mínimos requerimientos técnicos, una persona u organización puede crear sus contenidos sonoros y distribuirlos en Internet. En los últimos años, el podcasting ha iniciado una segunda era (Bonini, 2015) en la que redes de podcasts comercializan el patrocinio y la publicidad, y se crean empresas especializadas en la producción.

La evolución de podcasting supone también su irrupción en el ámbito de las relaciones públicas (Sellas, 2014), como una técnica singular con muchas posibilidades para la comunicación de las organizaciones. Y más allá de información corporativa, en los últimos años empresas como General Electric, eBay o Microsoft han lanzado podcasts de ficción o entretenimiento, un tipo de producto que de acuerdo con los principios de la theory of entertainment persuasion (Moyer-Gusé, 2008) tiene un notable potencial de impacto en los públicos. Aerolínea Momentos es un caso pionero en España.

\section{Metodología}

Para analizar el uso del podcasting en la comunicación corporativa de Iberia, se realizó una investigación cualitativa mediante un estudio de caso (Yin, 2014), método que se orienta al análisis de particularidades significativas y permite dar respuesta a preguntas sobre el cómo o el porqué de una determinada realidad. Esta investigación no es solo de carácter descriptivo, sino que pretende ofrecer una explicación del caso observado. El objeto de estudio es el podcast Aerolínea Momentos, producido por Podium Podcast (PRISA Radio) en colaboración con Iberia con motivo del 90 aniversario de la compañía aérea. El trabajo realizado pretende dar respuesta a las preguntas siguientes:

1. ¿Cómo se incorpora el podcasting en la campaña corporativa del 90 aniversario de Iberia?

2. ¿Por qué Aerolínea Momentos es un podcast apropiado en el marco de esa campaña?

Partiendo de estas cuestiones, se fijan los siguientes objetivos de investigación:

- O1. Analizar la integración del podcasting en la campaña corporativa para la celebración del 90 aniversario de Iberia

- O2. Exponer las características singulares del podcast Aerolínea Momentos y su aportación a la campaña corporativa.

Para abordar ese doble objetivo, se han utilizado diversas técnicas y procedimientos. En primer lugar, se ha llevado a cabo una investigación empírica secundaria (Soriano, 2007). Las fuentes consultadas (tabla 1) han sido de dos tipos: documentos corporativos de lberia y documentos periodísticos acerca de Iberia y su 90 aniversario (prensa y radio). Las fuentes y textos se han 
seleccionado a partir de los criterios de representatividad, autenticidad y credibilidad, que se estiman como apropiados en la investigación empírica secundaria (Deacon, Murdock, Pickering i Golding, 2007).

Se ha realizado un vaciado de estos documentos, textuales y sonoros, con una doble finalidad. Por un lado, conocer la identidad corporativa de lberia, así como los términos en los que se expresa por parte de la compañía y/o sus miembros. Se parte de un enfoque organizacional del concepto de identidad corporativa, que Capriotti (2009, p. 21) sintetiza como "el conjunto de características centrales, perdurables y distintivas de una organización, con las que la propia organización se identifica (a nivel introspectivo) y se autodiferencia (de las otras organizaciones de su entorno)". La identidad corporativa se nutre de dos componentes fundamentales: la cultura corporativa (entendida como aquello que la organización es) y la filosofía corporativa (aquello que quiere ser, que se expresa mediante la misión, la visión y los valores de la organización).

Por otro lado, la consulta documental pretendía conocer las principales acciones de lberia en su campaña de celebración del 90 aniversario, para identificar los mensajes de la compañía y la presencia de su identidad corporativa en la conmemoración de la efeméride.

Tabla 1. Fuentes documentales.

\begin{tabular}{|l|l|l|}
\hline Documento & Tipo & \multicolumn{2}{l|}{ Fuente } \\
\hline $\begin{array}{l}\text { Iberia - carpeta de prensa } \\
\text { (Iberia, 2018b) }\end{array}$ & Dosier de prensa & $\begin{array}{l}\text { Web corporativa de } \\
\text { Iberia }\end{array}$ \\
\hline Acerca de Iberia (Iberia, 2018a) & Página web & $\begin{array}{l}\text { Web corporativa de } \\
\text { Iberia }\end{array}$ \\
\hline $\begin{array}{l}\text { Informe de sostenibilidad 2016 } \\
\text { (Iberia, 2017a) }\end{array}$ & Memoria & $\begin{array}{l}\text { Web corporativa de } \\
\text { lberia }\end{array}$ \\
\hline $\begin{array}{l}\text { Conversaciones. La historia de Iberia } \\
\text { por quienes mejor la conocen }\end{array}$ & $\begin{array}{l}\text { Recopilatorio de } \\
\text { entrevistas }\end{array}$ & $\begin{array}{l}\text { Blog 'Me gusta volar' } \\
\text { de Iberia }\end{array}$ \\
\hline $\begin{array}{l}\text { Ronda. Edición especial 90 aniversario } \\
\text { de Iberia (Iberia, 2017b) }\end{array}$ & $\begin{array}{l}\text { Revista clase Bussines } \\
\text { i salas VIP }\end{array}$ & $\begin{array}{l}\text { Web corporativa de } \\
\text { Iberia }\end{array}$ \\
\hline $\begin{array}{l}\text { Iberia conecta pasado y presente } \\
\text { a través de experiencias de sus } \\
\text { empleados (EFE, 2017b) }\end{array}$ & $\begin{array}{l}\text { Crónica agencia EFE } \\
\text { (01/12/2017) }\end{array}$ & Web La Vanguardia \\
\hline
\end{tabular}




\begin{tabular}{|l|l|l|}
\hline $\begin{array}{l}\text { Podium Podcast e Iberia lanzan } \\
\text { Aerolínea Momentos. Juan Cierco, } \\
\text { dircom de Iberia; Miguel Martí, director } \\
\text { de Aerolínea Momentos; María Jesús } \\
\begin{array}{l}\text { Espinosa, jefa de proyecto de Podium } \\
\text { Podcast (Francino, 2017) }\end{array}\end{array}$ & $\begin{array}{l}\text { Conversación en el } \\
\text { programa 'La Ventana' } \\
(7 / 11 / 2017)\end{array}$ & Cadena SER \\
\hline $\begin{array}{l}\text { Nueve décadas surcando los cielos } \\
\text { (Núñez, 2017) }\end{array}$ & $\begin{array}{l}\text { Noticia El País } \\
(20 / 10 / 2017)\end{array}$ & El País digital \\
\hline $\begin{array}{l}\text { Iberia conmemora su 90 aniversario con } \\
\text { una exposición en el Centro Cultural } \\
\text { Conde Duque (Cadena SER, 2017) }\end{array}$ & $\begin{array}{l}\text { Noticia Cadena SER } \\
(19 / 10 / 2017)\end{array}$ & Web Cadena SER \\
\hline $\begin{array}{l}\text { Iberia celebra su 90 aniversario en la } \\
\text { 'MBFWMadrid' con una exposición de } \\
\text { sus uniformes históricos (Europa Press, } \\
\text { 2017) }\end{array}$ & $\begin{array}{l}\text { Noticia agencia Europa } \\
\text { Press (16/09/2017) }\end{array}$ & Web Europa Press \\
\hline $\begin{array}{l}\text { Iberia celebra su 90 aniversario con las } \\
\text { espectaculares maniobras de la Patrulla } \\
\text { Águila (EFE, 2017a) }\end{array}$ & $\begin{array}{l}\text { Noticia agencia EFE } \\
(25 / 06 / 2017)\end{array}$ & Web agencia EFE \\
\hline $\begin{array}{l}\text { Iberia: el ocaso y renacer de una marca } \\
\text { narrado por su dircom (Fernández, 2017) }\end{array}$ & $\begin{array}{l}\text { Artículo PR Noticias } \\
(05 / 05 / 2017)\end{array}$ & Web PR Noticias \\
\hline
\end{tabular}

Fuente: elaboración propia.

Paralelamente, se ha llevado a cabo un análisis de contenido del podcast Aerolínea Momentos. Se trata de un podcast original coproducido en 2017 por Iberia y Podium Podcast, la red de podcasts en español de PRISA Radio. Es una serie de ficción sonora que se inspira en la tradición de viajes en el tiempo para realizar un recorrido por los acontecimientos más importantes de las nueve décadas de vida de Iberia. La muestra la integran los nueve capítulos de la serie, que se detallan a continuación: 


\section{Tabla 2. Capítulos de Aerolínea Momentos (Podium Podcast, 2017)}

Capítulo Descripción de la trama (Podium Podcast)

1. Los prodigiosos 20

En la Real Academia de Bellas Artes de San Fernando Lorca, Dalí y Buñuel esperan a nuestros tres primeros pasajeros.

2. Infiernos y paraísos en los años 30

La línea de Iberia a las islas Baleares se abrió durante los años 30 y gente de toda España y parte de Europa aprovechó para cambiar el barco por el avión. Entre ellos, los miembros del Dadaísmo.

3. La aventura y el viaje en los 40

El nuevo vuelo de la Aerolínea Momentos se realiza en el interior de un clásico de la aviación: el Douglas DC3 en el que Hiato y la tripulación de la nave Kairós viajará a la Inglaterra de los años 40.

4. Amores secretos en los años 50

La comandante Anya Letts, la sobrecargo Ruth Vasán y el robhum Kirk están a punto de cambiar el rumbo de la historia. Cualquier pequeño cambio o modificación podría modificar todo.

5. Pop y flequillo en los años 60

La era hippy había entrado con fuerza en todo el mundo y la sobrecargo de la nave Kairós, Ruth Vasán, envidia aquellos años de música, baile y buenrollismo.

6. La década feminista de los 70

En este nuevo vuelo de la Aerolínea Momentos conoceremos a algunas mujeres que cambiaron el rumbo de la historia: Sor Juana Inés de la Cruz, Marie Curie, Virgina Woolf o Clara Campoamor.

7. Vaivenes mundiales en los años 80

El famoso cuadro Guernica de Picasso es el protagonista de este nuevo episodio en el que nos toparemos con los carismáticos Woody Allen y Tony Roberts con las manos en la masa...

8. Clones y robots en los años 90

Leo y Martina están de viaje de luna de miel, una luna de miel muy especial porque hacerlo a bordo de la nave Kairós siempre es diferente.

9. La primera década del siglo XXI

Mark Zuckerberg está a punto de cambiar el curso de la historia, pero él no lo sabe.

Fuente: elaboración propia, a partir de Podium Podcast (2017).

El instrumento de análisis es la matriz PodCred framework, un marco propuesto por Tsagkias, Larson y De Rijke (2010) para evaluar el potencial de un podcast a partir de una serie de indicadores que afectan a la percepción de los oyentes. Se agrupan en cuatro categorías:

1. Contenido: el tipo de contenido y su consistencia (tema, invitados, expertos, segmentación de la audiencia, trabajo de campo, información, opiniones, recomendaciones, testimonios y fuentes, entre otros).

2. Podcaster: locución, estilo y perfil del podcaster (cualidades acústicas, prosodia, pronunciación, vocabulario, personalidad o registro, entre otros).

3. Contexto: interactividad, participación (apelaciones, comentarios, respuestas, canales...) y contexto del podcast (profesional o amateur, publicidad, patrocinio...). 
4. Ejecución técnica: calidad de la producción (presentación, atmósfera, claridad, música, efectos de sonido, montaje técnico y expresivo) y empaquetado (metadatos, RSS, calidades, logo, imágenes).

PodCred Framework adopta los principios del entretenimiento y la investigación sobre la credibilidad e interés de los blogs para analizar los podcasts. Investigaciones internacionales que aplican este marco concluyen que sus indicadores son fiables para valorar la calidad e interés de un podcast y que permiten predecir de forma genérica una recepción favorable entre los oyentes (Paladino-Christin y Bétrancourt, 2016; Tsagkias, Larson y De Rijke, 2009, 2010; Waters, Ahmadullah, Bruun y Mathisen, 2012).

\section{La identidad corporativa y la celebración del 90 aniversario de Iberia}

El trabajo de campo realizado pretendía, en primer lugar, exponer la identidad corporativa de la empresa. Iberia se describe como una compañía aérea con 90 años de historia, en la que han volado 900 millones de clientes desde 1927, que es referencia entre Europa y América Latina y que cuenta con un total de 124 destinos en 48 países. La compañía destaca su transformación de los últimos años, que le permite hablar de una nueva Iberia, con una nueva cultura pensada con mayor atención al cliente, con nuevas formas de trabajo y más moderna y eficiente (Iberia, 2017a, 2018b). Capacidad reinventarse, afinidad con América Latina, talento y compromiso de los empleados, y puntualidad son claves de esta evolución, así como la sostenibilidad, tanto medioambiental como social.

Iberia define su misión como la de ofrecer servicios de transporte aéreo, aeroportuarios y de mantenimiento de aeronaves que den satisfacción a las expectativas de los clientes (Iberia, 2017a, 2017b, 2018a, 2018b). Junto a ello, remarca que sus actividades creen valor económico y social y que lo hagan de manera sostenible. La visión hace hincapié en el liderazgo, la satisfacción del cliente, la innovación, la rentabilidad económica y social. La misión y visión de lberia se sostienen sobre sus valores: enfoque al cliente, creación de valor, excelencia en la gestión, compromiso social, importancia de las personas, liderazgo, trabajo en equipo, mejora continua, adaptación al cambio, innovación y protección del medio ambiente.

A lo largo de 2017, Iberia ha conmemorado su 90 aniversario (Cadena SER, 2017; EFE, 2017a, 2017b; Europa Press, 2017). El 14 de diciembre de 1927, realizó el primer vuelo comercial desde Barcelona a Madrid. El avión aterrizó en el aeródromo Cuatro Vientos, donde tuvo lugar el acto inaugural de la compañía. 
Tabla 3. Actividades con motivo del 90 aniversario.

\begin{tabular}{|l|l|}
\hline Actividades de celebración del 90 aniversario & Fecha \\
\hline $\begin{array}{l}\text { Exposición retrospectiva en el Centro Cultural Conde Duque de Madrid, } \\
\text { en colaboración con la Agencia EFE }\end{array}$ & Octubre-diciembre 2017 \\
\hline Exposición de uniformes de Iberia en BMFW Madrid, Ifema & Septiembre 2017 \\
\hline Exhibición aérea en el aeródromo Cuatro Vientos de Madrid & $28 / 06 / 2017$ \\
\hline $\begin{array}{l}\text { 'Conversaciones' de empleados en la revista corporativa 'Ronda' } \\
\text { (distribuida en los vuelos) }\end{array}$ & Enero - diciembre 2017 \\
\hline Edición de libro recopilatorio de las 'Conversaciones' & Diciembre 2017 \\
\hline $\begin{array}{l}\text { Número especial de la revista corporativa Ronda (clase Business i salas VIP) } \\
\text { Distribución del número especial de Ronda en clase Turista }\end{array}$ & Junio 2017 \\
\hline $\begin{array}{l}\text { Acto con empleados y directivos en el Centro Cultural Conde Duque de } \\
\text { Madrid }\end{array}$ & $28 / 06 / 2017$ \\
\hline $\begin{array}{l}\text { Coproducción de Aerolínea Momentos, en colaboración con Podium } \\
\text { Podcast }\end{array}$ & \begin{tabular}{l}
$01 / 12 / 2017$ \\
\hline
\end{tabular} \\
\hline
\end{tabular}

Fuente: elaboración propia.

A partir del análisis de la documentación relativa a los actos del 90 aniversario, se han extraído las que se consideran ideas fuerza de la campaña vinculadas con la identidad de la compañía:

a) Valorar el pasado para mirar al futuro

Esta es una constante en las intervenciones del presidente de la compañía, Luis Gallego, y del dircom, Juan Cierco, tanto en los actos públicos como en medios de comunicación. También es el relato que sostiene la exposición '1927-2017. 90 años volando', que, a través de textos, objetos, imágenes (archivo EFE) y audiovisuales, muestra la historia de la aviación civil y de lberia y su impacto social. El monográfico de la revista Ronda aborda las claves del pasado (reportaje sobre el primer vuelo con descendientes de sus protagonistas) y el futuro (reportaje hacia los retos y oportunidades de la empresa, con los directores de Clientes, Comercial, Desarrollo de Red y Digital). Y del podcast Aerolínea Momentos, Cierco explica que la propuesta de Podium Podcast les permite vincular el pasado con una visión de futuro (Francino, 2017).

\section{b) Liderazgo e innovación}

Tanto en los documentos corporativos como en los actos públicos de la campaña, subyacen los valores de liderazgo e innovación de la compañía. Lo reconocen también las autoridades, como el ministro de Fomento, Íñigo de la Serna, quien en la inauguración de la exposición retrospectiva destaca el papel de Iberia en el transporte aéreo español, su capacidad para evolucionar en el tiempo y un papel decisivo en la contribución del progreso del país (Núñez, 2017). En Ronda, el presidente Luis Gallego insiste: "hemos puesto el foco en el área de Cliente, con la puntualidad como bandera, la nueva flota y reconfiguración de cabinas" (Iberia, 2017b, p. 62). La flota y su desarrollo a lo largo del tiempo está presente tanto en los documentos corporativos como en las actividades de conmemoración. 
La capacidad de innovación y mejora continua se puede observar en las exposiciones de la campaña. Por ejemplo, con los objetos que reflejan los cambios en la tecnología utilizada por Iberia. Pero también otras evoluciones de tipo social y cultural, como los audiovisuales que repasan la publicidad de lberia a lo largo de nueve décadas o la muestra de uniformes, espejo de cada época determinada. El liderazgo también pasa por la divulgación y la transparencia. Ese es el objetivo de '90 años, 90 cosas de Iberia y la aviación', uno de los cinco reportajes temáticos del número especial de Ronda. Otro reportaje muestra la historia de la compañía a través de la publicidad y un tercero es una foto historia de los hangares de mantenimiento.

El monográfico de Ronda, por otra parte, es una edición concebida como una obra de coleccionista. Incluye colaboraciones de profesionales como Chema Madoz, premio Nacional de Fotografía 2000 en España, quien fotografía la portada; la pintora Marina González Eme, quien ilustra diversos artículos; o el fotógrafo especializado en arquitectura, Juan Baraja, autor de las imágenes de los hangares. La calidad del servicio y del producto es un valor de lberia e intenta aplicarlo también a sus revistas corporativas.

\section{c) Marca España y vínculo especial con América Latina}

En la entrevista publicada en el monográfico de Ronda (Iberia, 2017b), Luis Gallego señala que "Iberia es una compañía muy española, muy asociada a la marca España. Llevamos nuestra cultura con orgullo y nos gusta que, cuando uno se sube en un avión, siente que pisa un trocito de España". Y añade: "Al recuperar rutas a países como Uruguay, República Dominicana o Cuba [...], uno se da cuenta de lo que significa Iberia en Latinoamérica. Hay una emoción que va mucho más allá de los negocios" (Iberia, 2017b, p. 62). Esa relación con la marca España aparece en otros momentos de la campaña, como la exposición de uniformes en BFMW Madrid, que permite al público ver los diseños que en su momento realizaron diseñadores como Pedro Rodríguez, Pertegaz, Elio Berhanyer, Alfredo Caral y Adolfo Domínguez, y que dieron a conocer la moda española en el mundo.

\section{d) El valor de las personas}

Poner en valor el compromiso y la dedicación de los empleados es uno de los ejes de la campaña del 90 aniversario. La celebración da protagonismo a los públicos internos. Lo argumenta el presidente de Iberia en 'Conversaciones': "hay algo -la verdadera esencia de lo que es Iberia- que ha seguido intacto, década tras década: la lealtad, el compromiso y la perseverancia de sus empleados" (Iberia, 2017b, p. 3). Ese protagonismo se concreta en los diálogos publicados en el blog corporativo a lo largo de 2017: 12 conversaciones, 24 personas, de distintos departamentos, un empleado actual que charla con otro que hacía sus mismas funciones en otra época. Personas con nombre y apellidos que explican su actividad profesional pero también muestran su lado más personal. Recopiladas en el libro 'Conversaciones', presentado en un acto público con los empleados de Iberia, el presidente actual Luis Gallego y el expresidente Xabier de Irala.

\section{Las claves de Areolínea Momentos como podcast}

El análisis de los nueve episodios de Aerolínea Momentos a través del PodCred Framework ofrece los siguientes resultados, de acuerdo con las cuatro categorías que plantea: 


\section{a) Contenido}

Aerolínea Momentos es una serie de ficción sonora de nueve capítulos y temporada única. La consistencia del contenido es una de sus fortalezas evidentes, tanto en el tema, como la trama y la estructura. El tema es el viaje en el tiempo. A partir de aquí, la trama, ambientada en el año 2150, se construye mediante los viajes de la nave Kairós, de la aerolínea espaciotemporal Momentos, al pasado. Cada capítulo está dedicado a una década. Los escenarios de cada episodio permiten revivir algunos de los principales hitos de Iberia a lo largo de su historia y las rutas que inauguró en cada una de las décadas.

La tripulación, integrada por la comandante Anya Letts, la sobrecargo Ruth Vasán y el robhum (robot con un porcentaje humano) Kirk, acompaña a los pasajeros (que varían según el episodio) a una década concreta, donde interactúan con algunos de los personajes clave del momento. Así, por ejemplo, coinciden con el rey Alfonso XIII en el primer vuelo de Iberia en los años 20, con los dadaístas en Ibiza en los 30, con Hemingway en la Cuba de los 40 o viajan a México en los 50 hasta la Casa Azul de Frida Kahlo y Diego Rivera, ven la llegada de los Beatles a Madrid en los 60, reviven el protagonismo de mujeres que cambiaron el mundo en los 70, tropiezan con Woody Allen en Nueva York en los 80, asisten a los Juegos Olímpicos de Barcelona 92 o viven en primera persona el discurso de Barack Obama al asumir la presidencia de los Estados Unidos en 2009.

La estructura de los episodios es siempre la misma: cuña de Iberia, indicativo de Podium Podcast, inicio del episodio, off introductorio de Kirk (que se repite, con leves variaciones, en todos los capítulos) sobre la sintonía de la serie, careta, desarrollo de la trama y resolución sobre música de la época determinada. Los tres protagonistas aparecen en todos los capítulos y otro elemento de cohesión es el rol de Kirk como relator de los principales acontecimientos políticos, sociales y culturales de cada década. Junto a ellos, en todos los episodios aparecen otros personajes (los pasajeros y algunos protagonistas de la época, ya sea con audios procedentes de fonoteca o bien mediante una interpretación del personaje en cuestión).

La serie cuenta con otros elementos que refuerzan la consistencia del contenido, de acuerdo con el marco PodCred. Se trata de una ficción sonora, pero está construida sobre la base de una investigación documental que queda reflejada en el guion. Junto a ello, se establecen elementos de conexión entre los distintos episodios, a partir del capítulo cuatro, mediante referencias a personajes o situaciones vividas en los anteriores. Finalmente, también cabe remarcar la regularidad en la duración de los episodios (entre 17 y unos 22 minutos), mientras que la frecuencia de emisión (entre el 19 de octubre de 2017 y el 21 de diciembre) es ligeramente más irregular.

\section{b) Podcaster}

En esta categoría, el marco PodCred considera elementos como la prosodia, el estilo, el lenguaje o las credenciales del podcaster. Y aquí, Aerolínea Momentos es una ficción sonora protagonizada por actores profesionales. Los tres personajes protagonistas los interpretan Antonio Dechent, Ingrid García-Jonsson y Macarena Gómez. El resto del reparto varía según el capítulo, pero siempre caracterizado por su profesionalidad. Incluso con guiños cinematográficos, como el personaje del detective que interpreta Ramón Langa (doblador de Bruce Willis) o Joan Pera en el papel de Woody Allen (al que dobla en cine). 
La locución de los profesionales y su interpretación es un gran trabajo, siempre con el registro necesario en función las necesidades de los personajes, de su situación y del momento de la trama. En el caso de los protagonistas, además, avanza de acuerdo con la propia evolución de los personajes. Cabe destacar aquí también la labor de los guionistas José Ángel Esteban y David Barreiro, así como del director Miguel Martí. El guión también permite cumplir con otro indicativo de esta categoría: la afiliación del podcaster. A lo largo de los episodios, aparecen numerosas referencias a Iberia en boca de los protagonistas, aspecto que refuerza la identificación del producto con la marca.

\section{c) Contexto}

Esta categoría hace referencia a la relación del podcast con los oyentes, así como la presencia de material adicional y la creación de comunidad. Es el apartado en el que Aerolínea Momentos muestra algunas debilidades. Así, el podcast no dispone de perfil específico en redes sociales, sino que aparece en el genérico Podium Podcast. La interacción con los oyentes también debe realizarse a través de los perfiles de Podium Podcast y los comentarios corren el riesgo de verse superados por muchos otros posts de otros programas que forman parte este portal de podcasting. Por su parte, los perfiles de Iberia en redes sociales difunden también los contenidos. Aerolínea Momentos es un producto de calidad que complementa una campaña, más que un canal para dialogar con los públicos.

\section{d) Ejecución técnica}

Esta categoría hace referencia a indicadores que permiten valorar la calidad del podcast en tanto que producto sonoro. Aerolínea Momentos sobresale de nuevo, gracias a la labor de Pablo Arévalo, técnico de Cadena SER y Podium Podcast, en la realización sonora. La ejecución técnica demuestra la optimización de la sintaxis sonora para la construcción del espacio, el tiempo y el ritmo. En este sentido, el podcast pone en valor la narrativa radiofónica y el montaje técnico y expresivo. Guionistas y realizador utilizan de forma óptima los elementos del lenguaje radiofónico (palabra, música, efectos de sonido y silencio), en sus diversas funciones (descriptiva, expresiva, narrativa y gramatical) para intentar la reconstrucción de cada momento a partir del sonido.

En cuanto al empaquetado del podcast, Aerolínea Momentos dispone de su propio espacio dentro de la web de Podium Podcast, aunque la ruta de acceso requiere de algunos pasos intermedios. Una vez en su página, el acceso a los episodios es claro, en sus distintas modalidades (streaming, descarga, iTunes, Ivoox y RSS). El podcast dispone también de una imagen gráfica distintiva.

\section{Aerolínea Momentos en la estrategia de Iberia}

Iberia desarrolla desde 2014 una nueva política enfocada primero a las realidades (pérdida de rutas históricas, malos resultados económicos, conflictividad laboral) para pasar posteriormente a las percepciones (Fernández, 2017). La iniciativa del podcast de ficción Aerolínea Momentos se enmarca en esta segunda fase estratégica. El proyecto se hace realidad en un contexto de resurgimiento de los géneros ficcionales sonoros.

Mediante la fórmula del viaje en el tiempo, a partir de un lejano futuro, esta ficción sonora permite repasar los principales acontecimientos de la historia de la aerolínea (muy vinculada 
a la propia evolución de la sociedad española), poner de relieve los fundamentos de la empresa y ofrecer a los públicos un producto coherente con valores corporativos de lberia como la innovación, el liderazgo, la creación de valor, la mejora continua o la adaptación al cambio. Aerolínea Momentos es una compañía que ofrece viajes a medida, que permite disfrutar del momento. Su tripulación se preocupa por el bienestar de los pasajeros, trabaja en equipo y es la mejor valorada en las encuestas de satisfacción. Siempre, además, llega a su destino con puntualidad. Con sentido del humor, con sentimientos, personajes con los que el oyente empatiza. En un futuro muy distinto al presente, como consecuencia de los daños sufridos por el medio ambiente: el guion incluye mensajes de responsabilidad social.

Desde una perspectiva estratégica, Aerolínea Momentos es en el fondo un trasunto de Iberia. Para Iberia, el podcast es una oportunidad para hacer algo distinto en su política de comunicación. Una licencia para mirar atrás mediante la ficción sonora, y al mismo tiempo, observar el futuro. Lo apunta el mismo Kirk al final del primer capítulo: "por eso queríamos terminar el viaje de esta manera, para que aprendáis lo importante que es aprender del pasado para preparar el futuro" (Podium Podcast, 2017).

Así como el número especial de la revista corporativa Ronda está pensado como un ejemplar de colección, también Aerolínea Momentos es un producto singular. Una ficción sonora que se entiende en el contexto de una campaña determinada: la del 90 aniversario de Iberia. Un branded podcast, además, que cumple con los principales indicadores del PodCred framework, como son la calidad del contenido, la coherencia con la identidad corporativa, la excelencia del podcaster y la optimización del lenguaje sonoro.

\section{Conclusiones}

El uso del podcasting en las relaciones públicas es poco habitual. Las experiencias significativas se han llevado a cabo en el ámbito anglosajón, mientras que en España existen pocos precedentes (Sellas, 2014). El caso del podcast Aerolínea Momentos en la campaña del 90 aniversario de Iberia resulta singular: un branded podcast basado en una ficción sonora por capítulos.

El análisis llevado a cabo refleja la integración estratégica de Aerolínea Momentos en la celebración del 90 aniversario de Iberia. El podcast forma parte de una campaña que, como se ha detallado, contaba con diferentes iniciativas que no solo pretendían conmemorar el nacimiento de la compañía y el inicio de los vuelos, sino remarcar su aportación a la evolución social y económica de España. En este contexto, la ficción sonora Aerolínea Momentos pone en valor la historia de la compañía, a la vez que integra, expone y refuerza los aspectos clave de la identidad corporativa de Iberia.

Por otro lado, Aerolínea Momentos es un proyecto de calidad realizado por especialistas con el conocimiento y las competencias necesarios para ocuparse de los podcasts de las organizaciones. Como producto sonoro, responde a los parámetros que le otorgan potencial para generar un impacto en los públicos. Como herramienta de identidad corporativa, permite a la dirección de comunicación de lberia incorporar elementos innovadores en la campaña de celebración del 90 aniversario. 
Aerolínea Momentos es un caso pionero en España y sigue la estela de algunos precedentes de éxito en Estados Unidos, como los branded podcast de General Electric. Ampliar el abanico de casos analizados y realizar una comparativa internacional son nuevas investigaciones con las que se pretende dar continuidad al camino iniciado con el presente artículo. El podcast de ficción abre una nueva vía para la creatividad y la innovación en la comunicación de las organizaciones. Pero el podcasting tiene también algunos inconvenientes, como su naturaleza unidireccional o la necesidad de canales complementarios que permitan el diálogo entre la organización y sus públicos. Como se pudo observar en la revisión de literatura académica realizada, el impacto de las TIC y los medios sociales en las relaciones públicas plantea todavía muchas cuestiones pendientes.

\section{Referencias bibliográficas}

[1] Bonini, T. (2015). La 'segona era' del podcàsting: el podcàsting com a nou mitjà de comunicación de masses digital. Quaderns del CAC, 18(41), 23-33.

[2] Cadena SER (2017, octubre 19). Iberia conmemora su 90 aniversario con una exposición en el Centro Cultural Conde Duque. Cadena SER. Consultado 28 febrero 2018, desde http://cadenaser.com/emisora/2017)10/19/radio madrid/1508429066 476088.html

[3] Capriotti, P. (2009). Branding corporativo. Santiago de Chile: Colección de libros de empresas.

[4] Daniels, Ch. (2017, septiembre 29). Branded podcasts make a comeback. PR Week. Consultado 28 febrero 2018, desde https://www.prweek.com/article/1446083/ branded-podcasts-comeback

[5] Deacon, D., Murdock, G., Pickering, M., Golding, P. (2007). Researching communications. A practical guide to methods in media and cultural analysis ( $2^{\text {nd }} e d$.). London: Bloomsbury Academic.

[6] Duhé, S. (Ed.) (2007). New media and public relations. New York: Peter Lang.

[7] Duhé, S. (2015). An overview of new media research in public relations journals from 1981 to 2014. Public Relations Review, 41(2), 153-169.

[8] EFE (2017a, junio 26). Iberia celebra su 90 aniversario con las espectaculares maniobras de la Patrulla Águila. ABC. Consultado 28 febrero 2018, desde https://www.abc.es/ economia/abci-iberia-celebra-90-aniversario-espectaculares-maniobras-patrullaaguila-201706251816 noticia.html

[9] EFE (2017b, diciembre 1). Iberia conecta pasado y presente a través de experiencias de sus empleados. La Vanguardia. Consultado 28 febrero 2018, desde https://www. lavanguardia.com/vida/20171201/433335810310/iberia-conecta-pasado-y-presente-atraves-de-experiencias-de-sus-empleados.html

[10] Estanyol, E. (2012). Marketing, public relations, and how Web 2.0 is changing their relationship. A qualitative assessment of PR consultancies operating in Spain. Public Relations Review, 38(5), 831-837.

[11] Europa Press (2017, septiembre 16). Iberia celebra su 90 aniversario en la 'MBFWMadrid' con una exposición de sus uniformes históricos. Europa Press. Consultado 28 febrero 2018, desde http://www. europapress.es/madrid/ifema-00444/noticia-iberia-celebra90-aniversario-mbfwmadrid-exposicion-uniformes-historicos-20170916174814.html

[12] Fernández, G. (2017). Iberia: el ocaso y renacer de una marca narrado por su dircom. Consultado 18 diciembre 2017, desde https://prnoticias.com/comunicacion/ prcomunicacion/20162286-iberia-caso-exito 
[13] Fernández-Quijada, D. y Ramos-Serrano, M. (Eds.) (2014). Tecnologías de la persuasión. Uso de las TIC en publicidad y relaciones públicas. Barcelona: UOC.

[14] Francino, C. (Locutor. 2017, noviembre 7). Aerolínea Momentos [Audio Podcast]. Consulta 8 noviembre 2017, desde http://play.cadenaser.com/audio/cadenaser laventana $20171107190000200000 /$

[15] Iberia. (2017a). Informe de sostenibilidad Iberia 2016. Consultado 22 febrero 2018, desde http://grupo.iberia.es/portal/site/WebCorporativa/ menuitem.9f6f76b37fbac6bd5c3bd55cf34e51ca

[16] Iberia. (2017b). Conversaciones. Consultado 26 febrero 2018, desde http://megustavolar. iberia.com/wp-content/uploads/PDF-Libro-CONVERSACIONES-90-final-2.pdf

[17] Iberia. (2018a). Acerca de Iberia. Consultado 22 febrero 2018, desde http://grupo.iberia. es/portal/site/WebCorporativa/menuitem.098efbddfde1334ac4c39b10f34e51ca/

[18] Iberia.(2018b). Iberia - carpeta de prensa. Consultado 24 febrero 2018, desde http://grupo. iberia.es/portal/site/WebCorporativa/menuitem.b4484eccffd4134ac4c39b10f34e51ca/

[19] McCallister-Spooner, S.M. (2009). Fulfilling the dialogic promise. A ten-year reflective survey on dialogic Internet principles. Public Relations Review, 35(3), 320-322.

[20] Moyer-Gusé, E. (2008). Toward a theory of entertainment persuasion: Explaining the persuasive effects on entertainment education messages. Communication Theory, 18(3), 407-425.

[21] Núñez, J. (2017). Nueve décadas surcando los cielos. Una muestra repasa la historia de iberia en su 90 aniversario. Consulta 15 diciembre de 2017, desde https://elpais.com/ ccaa/2017/10/19/madrid/1508435571_213125.html

[22] Paladino-Christin, M. y Bétrancourt, M. (2016). Usages et préférences de design concernant les podcasts audio professionnels : enquête des professionales des TIC. Revue internationale des technologies en pédagogie universitaire, 13(2-3), 46-59.

[23] Peinado-Miguel, F., Rodríguez-Barga, D. y Rodríguez-Pallares, M. (2017). Hacia un nuevo modelo de negocio radiofónico: la radio corporativa. Estudios sobre el Mensaje Periodístico, 23(1), 147-164.

[24] Piñeiro-Otero, T. (2015). Del jingle a las radios corporativas. Una aproximación al concepto de audiobranding. Prisma Social, (14), 663-688.

[25] Podium Podcast. (2017). Aerolínea Momentos. Consultado 10 febrero 2018, desde http:// www.podiumpodcast.com/aerolinea-momentos/

[26] Prata, N. y Cordeiro, H. (2017). Brand radio: La segmentación personalizada como modelo de negocio. Revista de la Asociación Española de Investigación de la Comunicación, 4(7), 77-85.

[27] Sánchez, F. (2010). Radio corporativa: la radio del futuro. Consultado 2 de marzo 2018, desde https://www.panoramaaudiovisual.com/2010/09/28/radio-corporativa-laradio-del-futuro/

[28] Sellas, T. (2014). "El podcasting en las relaciones públicas: la voz de las organizaciones en la web 2.0". En Fernández-Quijada, D. y Ramos-Serrano, M. (Eds.) (2014). Tecnologías de la persuasión. Uso de las TIC en publicidad y relaciones públicas. Barcelona: UOC, 197-214.

[29] Soriano, J. (2007). L'ofici de comunicòleg. Mètodes per investigar la comunicació. Barcelona: Eumo.

[30] Taylor, M. y Kent, M. (2010). Anticipatory socialization in the use of social media in public relations: A content analysis of PRSA's Public Relations Tactics. Public Relations Review, 36(3), 207-214. 
[31] Tsagkias, M., Larson, M. y de Rijke, M. (2009). Exploiting surface features for the prediction of podcast preference. In $31^{\text {st }}$ European Conference on Information Retrieval Resarch (ECIR 2009), Toulouse, 6-9 de abril de 2009 (pp. 473-484). Berlin: Springer.

[32] Tsagkias, M., Larson, M. y de Rijke, M. (2010). Predicting podcast preference: An analysis framework and its application. Journal of the American Society for Information Science and Technology, 61, 374-391.

[33] Verčič, D., Verčič, A.T. y Siramesh, K. (2015). Looking for digital in public relations. Public Relations Review, 41(2), 142-152.

[34] Waters, R.D., Ahmadullah, A., Bruun, L. y Mathisen, K.S. (2012). Messaging, musica, and mailbags: How technical design and entertainment boost the performance of environmental organizations' podcasts. Public Relations Review, 38(1), 64-68.

[35] Yin, R.K. (2014). Case study research and methods ( $5^{\text {th }}$ ed.). Thousand Oaks: Sage.

\section{CURRICULUM VITAE. Toni Sellas}

Toni Sellas es Profesor Agregado del Departamento de Comunicación de la Universitat de Vic - Universitat Central de Catalunya. Docente e investigador en la Facultad de Empresa y Comunicación de la UVic - UCC. Licenciado en Ciencias de de la Información por la Universidad de Navarra y Doctor en Ciencias Humanas, Sociales y Jurídicas por la Universitat Internacional de Catalunya (UIC). Sus principales líneas de investigación son el podcasting, la radio online y la evolución de la industria radiofónica. En 2009 defendió la primera tesis doctoral en España sobre el podcasting, temática sobre la que ha publicado en revistas académicas nacionales e internacionales, además de monografías y capítulos en obras colectivas. Colabora como revisor con diversas publicaciones académicas. Es miembro de Podcast Studies Network, de la Asociación Española de Investigadores en Comunicación (AE-IC) y de la European Communication Reseach and Education Association (ECREA). 